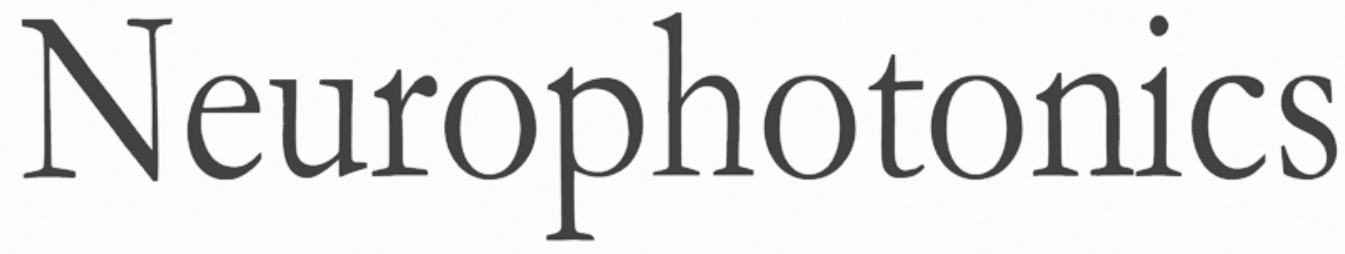

\title{
Characterization of a fiber-less, multichannel optical probe for continuous wave functional near- infrared spectroscopy based on silicon photomultipliers detectors: in-vivo assessment of primary sensorimotor response
}

\author{
Antonio M. Chiarelli \\ Sebania Libertino \\ Filippo Zappasodi \\ Massimo Mazzillo \\ Francesco Di Pompeo \\ Arcangelo Merla \\ Salvatore Lombardo \\ Giorgio Fallica
}




\title{
Characterization of a fiber-less, multichannel optical probe for continuous wave functional near-infrared spectroscopy based on silicon photomultipliers detectors: in-vivo assessment of primary sensorimotor response
}

\author{
Antonio M. Chiarelli, ${ }^{a, \star}$ Sebania Libertino, ${ }^{b}$ Filippo Zappasodi, ${ }^{a}$ Massimo Mazzillo, ${ }^{c}$ Francesco Di Pompeo, ${ }^{a}$ \\ Arcangelo Merla, ${ }^{a}$ Salvatore Lombardo, ${ }^{b}$ and Giorgio Fallica ${ }^{c}$ \\ ${ }^{a}$ G.d'Annunzio University of Chieti-Pescara, Institute for Advanced Biomedical Technologies, Department of Neurosciences, \\ Imaging and Clinical Sciences, Italy

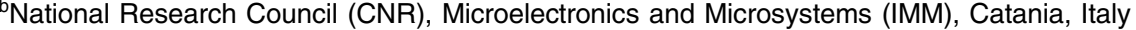 \\ 'STMicroelectronics, Research and Development, Catania, Italy
}

\begin{abstract}
We report development, testing, and in vivo characterization of a multichannel optical probe for continuous wave (CW) functional near-infrared spectroscopy (fNIRS) that relies on silicon photomultipliers (SiPMs) detectors. SiPMs are cheap, low voltage, and robust semiconductor light detectors with performances analogous to photomultiplier tubes (PMTs). In contrast with PMTs, SiPMs allow direct contact with the head and transfer of the analog signals through thin cables greatly increasing the system flexibility avoiding optical fibers. The coupling of SiPMs and light-emitting diodes (LEDs) made the optical probe lightweight and robust against motion artifacts. After characterization of SiPM performances, which was proven to provide a noise equivalent power below $3 \mathrm{fW}$, the apparatus was compared through an in vivo experiment to a commercial system relying on laser diodes, PMTs, and optical fibers for light probing and detection. The optical probes were located over the primary sensorimotor cortex and the similarities between the hemodynamic responses to the contralateral motor task were assessed. When compared to other state-of-the-art wearable fNIRS systems, where photodiode detectors are employed, the single photon sensitivity and dynamic range of SiPMs can fully exploit the long and variable interoptode distances needed for correct estimation of brain hemodynamics using CW-fNIRS. ๑ 2017 Society of PhotoOptical Instrumentation Engineers (SPIE) [DOI: 10.1117/1.NPh.4.3.035002]
\end{abstract}

Keywords: continuous wave functional near-infrared spectroscopy; silicon photomultipliers; primary sensorimotor response; wearable neuroimaging.

Paper 17095RR received May 9, 2017; accepted for publication Sep. 1, 2017; published online Sep. 27, 2017.

\section{Introduction}

Near-infrared (NIR) light can be applied to investigate human brain functional activity (functional near-infrared spectroscopy, fNIRS). ${ }^{1,2}$

fNIRS measures NIR (650 to $950 \mathrm{~nm}$ ) light that travels through the brain tissue and it estimates fluctuations in the concentration of oxy- $\left(\mathrm{O}_{2} \mathrm{Hb}\right)$ and deoxyhemoglobin $(\mathrm{HHb})$ in the cortex. ${ }^{3}$ These fluctuations are related to neural activity through the neurovascular coupling mechanism [blood oxygen level dependent (BOLD) effect] ${ }^{4} \mathrm{O}_{2} \mathrm{Hb}$ and $\mathrm{HHb}$ are the main chromophores that fluctuate in the NIR spectral range and they provide different absorption spectra. These characteristics, together with the water's low absorption and the high diffusive properties of biological tissues, allow estimating $\mathrm{O}_{2} \mathrm{Hb}$ and $\mathrm{HHb}$ concentration, and oscillations employing light sources and detectors located on the same investigation surface (the scalp, backscattering geometry ${ }^{5}$ ).

In the last two decades, fNIRS has become a diffused brain imaging modality, suited for investigation in multiple populations and experimental conditions. ${ }^{6-17}$

*Address all correspondence to: Antonio M. Chiarelli, E-mail: antonio.chiarelli@ unich.it
fNIRS has multiple advantages when compared to other neuroimaging modalities that measure brain hemodynamics, such as functional magnetic resonance imaging (fMRI) and positron emission tomography (PET). fNIRS has cheap hardware, it is robust against motion artifacts, it does not impose major physical constraints, and it does not involve exposure to high-magnetic fields and/or ionizing radiations.

Different technologies have been developed for fNIRS. Three main classes can be identified: time-domain (TD) ${ }^{18}$ frequency-domain (FD), ${ }^{19}$ and continuous wave $(\mathrm{CW})^{20}$ recording systems. TD systems use very short pulses of light (of the order of few picoseconds) and they can measure the distribution of photons' arrival time. TD instruments provide the highest amount of tissue optical properties information (both absolute and differential). However, these instruments are very expensive and are, therefore, generally limited to only a few source-detector pairs, severely limiting brain coverage capabilities. FD systems use light modulated at radio frequencies $(>50 \mathrm{MHz})$. They can provide information about the optical properties of the tissue (both absolute and differential) ${ }^{19}$ by measuring light attenuation and phase delays. However, they are more expensive and 
technologically complex than CW systems. CW systems measure the $\mathrm{CW}$ component of light that traveled through the investigated tissue. CW systems provide estimates of hemoglobin and oxygenation changes over time but they do not provide absolute estimation of tissue optical properties. However, because of the cheap and simple characteristic of this technology, and the focused interest in hemoglobin fluctuations for functional brain imaging, they are the most common systems by and large.

fNIRS measures are sensitive to optical phenomena occurring within volumes that have approximately the shape of curved spindles ("bananas"), whose size and position in the head is determined by the location of light sources and detectors on the scalp, as well as the high diffusive optical properties of the head structures. ${ }^{21}$ Because light sensitivity decays exponentially (within few $\mathrm{cm}$ ) from the given source-detector couple (channel), fNIRS provides good localization capabilities. However, multiple optodes (sources and detectors) must be employed in order to investigate the region of interest and to create a reasonable coverage of the brain cortex. Moreover, high-density, multiple source-detector distances, optical arrays (with distances ranging from $\sim 1.5 \mathrm{~cm}$ up to $\sim 6 \mathrm{~cm}$ ), must be employed in order to discriminate between intra- and extracerebral (scalp-related) hemoglobin oscillations ${ }^{22,23}$ and to create volumetric functional images [diffuse optical tomography (DOT)]. ${ }^{21,24-26}$

This implies that for both channel-based fNIRS and DOT fNIRS, a high numerosity, multidistance optodes configuration is required in order to achieve a good sensitivity, localization power, and cortex "field of view."

Typically, fNIRS measurements are performed by placing optical fibers on the skin for signal delivery and collection. Optical fibers are then connected to sources and sensitive detectors. Optical fibers are useful since they can be used to electrically isolate the subject, or whenever the fNIRS instrument must be placed at a certain distance (e.g., when performing simultaneous fMRI and fNIRS measurements). However, optical fibers introduce constraints to fNIRS. When employing optical fibers, it is difficult to keep a stable optode to scalp coupling and to dampen the effect of movement artifacts and ambient light. Moreover, a high number of fibers and fiber bundle ${ }^{27}$ (the bundles are required to detect enough light from the head surface) cause a loss in the lightweight properties of fNIRS reducing flexibility and often restricting the measurements to a laboratory environment.

Indeed, the ideal solution should be to place the detector directly in contact with the skin in order to avoid the use of optical fiber. ${ }^{28}$ State-of-the-art wearable CW-fNIRS systems employ photodiodes for light detection, however, their sensitivity and dynamic range are poor and these systems are generally limited to a few sparse optodes with fixed source-detector distances. ${ }^{29-31}$

In fact, the use of sensitive detectors, such as photomultiplier tubes (PMTs), does not allow locating detectors directly on the scalp.

The use of PMTs is impractical in real-life operations since they are bulky, fragile, and operate at high voltages. Recently, high-sensitivity solid-state detectors, such as single-photon avalanche diodes, have been applied for fNIRS but this solution is not optimal since the detector area is very small.

In the last year, silicon photomultipliers (SiPMs), initially developed as a photon-number resolving detector for highenergy physics applications (e.g., PET scanners, Cherenkov telescopes), ${ }^{32}$ have been proposed for biomedical applications ${ }^{33}$ including fNIRS. ${ }^{34-36}$ SiPMs are pixelated photodetectors of APDs working in Geiger mode. ${ }^{37}$ Compared to other semiconductor photodetectors, SiPMs present major advantages of sensitivity, high internal gain, and speed of response. ${ }^{32,38,39} \mathrm{SiPMs}$ provide a much higher responsivity, $\sim 3$ to $\sim 5.5$ orders of magnitude, ${ }^{36}$ than standard photodiodes or APDs. Compared to PMTs, SiPMs are much more compact and easy to handle, have much lower operating voltage, and are mechanically robust, optically resistant, and electrically reliable.

Here, we present a developed multichannel, multidistance, source time-multiplexed, CW-fNIRS device that relies on an optical probe constituted of three SiPM detectors and four LEDS sources (two injection points, two sources for each light injection location at 735 and $850 \mathrm{~nm}$ wavelengths). The optical probe is similar to that of an EEG system, where many probes (SiPM detectors and LED sources instead of electrodes) are located on the head and the analog signal is transferred via thin cables to a benchtop acquisition system.

Although the probe provided a limited number of optodes, it encompassed the required characteristics of a SiPMs-based CW-fNIRS system and it can be easily expanded. The sources and detectors were located directly on the scalp in a multidistance configuration. The overall optical probe covered $30 \mathrm{~cm}^{2}$. The system was first characterized, and its linearity and signalto-noise ratio (SNR) capabilities were addressed. The system was further tested in vivo on a subject undergoing a motor task (right hand finger tapping). The probe was located over the contralateral primary sensorimotor cortex and the retrieved hemoglobin changes were compared with results obtained using a commercial system (ISS Imagent ${ }^{\mathrm{TM}}$, Champaign, Illinois) and an identical optical probe geometry. The commercial system employed laser diodes and multimode fibers for light injection, and fiber bundles and high voltage PMTs for light detection.

\section{Methods}

\subsection{System Setup}

\subsubsection{Silicon photomultipliers and light-emitting diodes}

Large area N-on-P SiPM detectors manufactured at STMicroelectronics clean room facilities were employed [Fig. 1(a)]. The devices were fabricated on p-type silicon epitaxial wafers and formed of $n^{+}-p$ microcells. The quenching resistor, made from low-doped polysilicon, was integrated inside the cell. Thin optical trenches filled with oxide and metal surrounded the microcell active area in order to reduce the electrooptical coupling effects (crosstalk) between adjacent microcells. Details on the manufacturing method of STMicroelectronics SiPM technology are reported elsewhere. ${ }^{40,41}$ Even if not yet commercialized, ST SiPM performances are comparable to those of similar technologies available on the market ${ }^{42-44}$ in terms of gain, photon detection efficiency (PDE), and dark noise rate. N-on-P SiPMs were chosen for CW-fNIRS application because of their higher PDE in visible-NIR wavelength ranges when compared to the $\mathrm{P}$-on-N version of the technology. ${ }^{40,45} \mathrm{SiPMs}$ had a total area of $4.0 \times 4.5 \mathrm{~mm}^{2}$ and 4871 square microcells with $60-\mu \mathrm{m}$ pitch. The devices had a geometrical fill factor of $67.4 \%$ and were packaged in a surface mount housing (SMD) with $5.1 \times 5.1 \mathrm{~mm}^{2}$ total area. The SMD package was sealed by an epoxy resin transparent to the visible and NIR light with a refractive index of about 1.5 at room temperature. An Edmund Optics optical cast plastic CR-39 ${ }^{\circledR}$ (Allyl Diglycol Carbonate) NIR long-pass filter with a refraction 
(a)

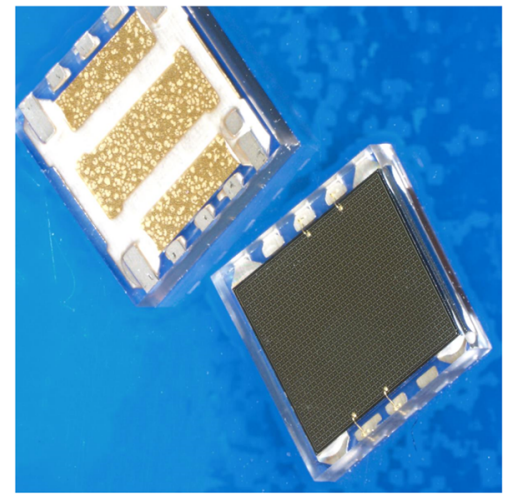

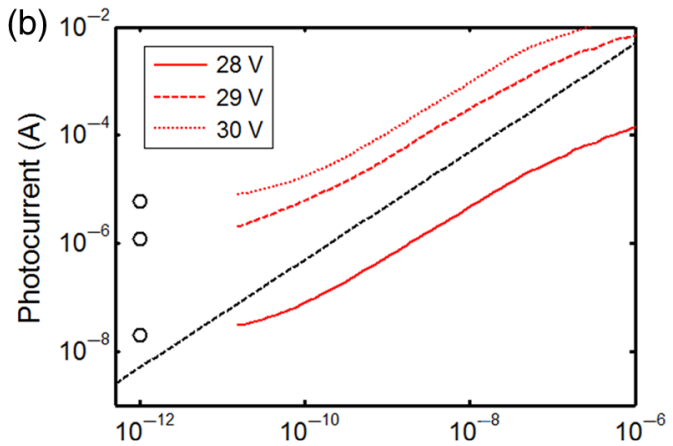

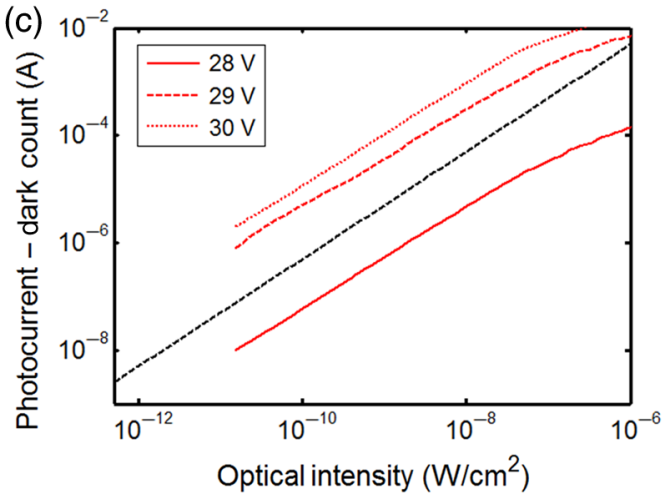

Fig. 1 (a) Example of two large area N-on-P SiPM detectors manufactured at ST (front and back view). The devices are packaged in a surface mount device (SMD) housing with $5.1 \times 5.1 \mathrm{~mm}^{2}$ total area. (b) SiPMs photocurrent as a function of optical intensity measured at $25^{\circ} \mathrm{C}$. Dark count levels and photocurrents at three bias voltages are reported as open circles, and as continuous, dashed, and dotted red lines, respectively. The ideal linear trend is indicated by the dashed black line. (c) SiPMs photocurrents after subtraction of the dark count rate. Photocurrents are reported as continuous, dashed, and dotted red lines. The ideal linear trend is indicated by the dashed black line.

index of 1.5 at $20^{\circ} \mathrm{C}, 700 \mathrm{~nm}$ cut-on wavelength, and an optical transmission higher than $90 \%$ in NIR range was glued on the SMD package by using a Loctite ${ }^{\circledR} 352^{\mathrm{TM}}$ adhesive. The NIR filter reduced the absorption of environmental light of more than $85 \%$ in the linear operation range of the detector, thus consistently increasing SiPMs' sensitivity, especially for very weak photon fluxes. Moreover, the use of the filter produced a considerable decrease of the SiPM dark current ${ }^{46}$ due to the reduction of optical cross talk effects triggered by secondary photons emitted in the visible range. Both these effects resulted in a higher SiPM SNR in a wider range of operation conditions, including high operation bias. With the filter, a maximum PDE of about $25.5 \%$ was measured at $735 \mathrm{~nm}$ and 3 V overvoltage above the SiPM breakdown voltage $(\sim 27 \mathrm{~V})$. A residual PDE lower than $0.1 \%$ was measured below $600 \mathrm{~nm}$, confirming the good light blocking properties of the filter in the visible range. ${ }^{47}$ Epitex bi-color SMT735 AlGaAs LEDs in SMD package emitting at 735 and $850 \mathrm{~nm}$ wavelengths were employed as optical light sources. The LEDs had an area of $2.6 \times 4.5 \mathrm{~mm}^{2}$, viewing angle of $55 \mathrm{deg}$, average spectral bandwidth of $20 \mathrm{~nm}$ at $735 \mathrm{~nm}$ and $35 \mathrm{~nm}$ and $850 \mathrm{~nm}$ emission wavelength. The average power emission ranged from 1 to $20 \mathrm{~mW}$. Both the SiPMs and LEDs boards were electrically isolated through plastic containers, resin, and optical filters.

\subsubsection{Optical probe and system architecture}

SiPMs were mounted on a small PCB, about $1 \mathrm{~cm}^{2}$ large [Fig. 2(a)], containing only the detector and passive components (a $1-\mathrm{k} \Omega$ sensing resistor and a $100-\mathrm{nF}$ filter capacitor). These small boards were connected to the acquisition electronics with flat cables $2 \mathrm{~m}$ long. Similarly, each dual wavelength LED was mounted on a small PCB, with the same connection layout [Fig. 2(a)]. The boards were glued on a piece of dark

(a)

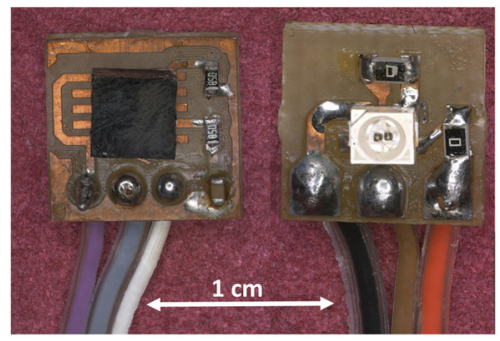

(b)

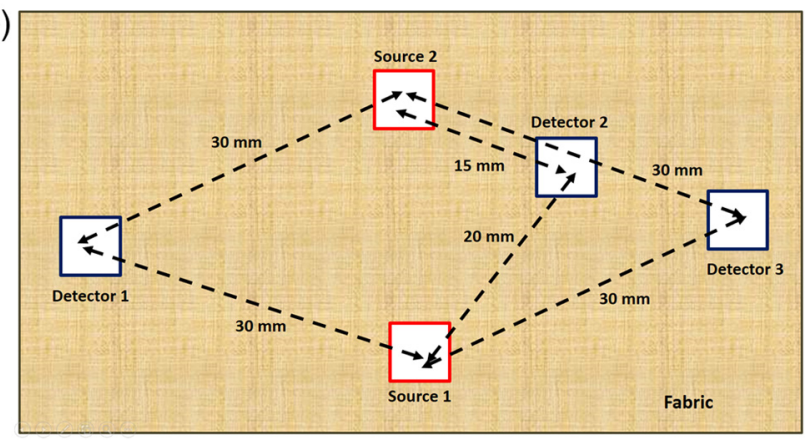

Fig. 2 (a) Example of a SIPM and a LED mounted on the small boards that were connected to the acquisition system and that were employed in the final dark-fabric optical probe. (b) Schematic representation of the optodes' layout employed. 
fabric with an optode layout reported in Fig. 2(b). In order to have a probe with a smooth front surface, a sheet of black rubber was applied, with suitable holes in correspondence with sources and detectors. The thickness of the rubber, even compressed, was higher than those of LEDs and SiPMs, to assure optical isolation between sources and detectors. This very simple probe was highly flexible and comfortable.

The electrical scheme is shown in Fig. 3. The LEDs were connected, through resistors, to a function generator providing a multiplexed square pulse (left side of Fig. 3). SiPMs (right side of Fig. 3) were connected to a RC integrator. The integrator time constant was chosen as $0.15 \mathrm{~ms}$ to be in the range of the recording oscilloscope sampling frequency of $10 \mathrm{kHz}$. The RC integrator acted as a low-pass filter rejecting high frequency ( $\gg 10 \mathrm{kHz}$ ) signals across the $R_{1,2}$ resistors to the oscilloscope input. Measured SiPMs photocurrent was in the range between 5 and $50 \mu \mathrm{A}$, which corresponded (with $R_{1}=R_{2}=15 \mathrm{k} \Omega$ ) to a signal in the range 0.1 to $1 \mathrm{~V}$, easily measured by the digital oscilloscope (Tektronix TPO 7500, $1 \mathrm{GHz}$ ).

LEDs were switched on and off at a frequency of $20 \mathrm{~Hz}$. One phase was dedicated to the dark count recording. For each phase, in which either only one color LED was on and all the others were off, or the LEDs were all off for dark current measurement, the same time interval was used. The time dedicated to each phase per cycle was $10 \mathrm{~ms}$. SiPMs were biased with the cathode at $+30 \mathrm{~V}$ while the anode was connected to the filtering circuit described above. The SiPMs' signals recorded by the oscilloscope were averaged in each phase of the cycle, i.e., in 10-ms time intervals.

\subsection{System Characterization}

SiPM devices, as described in Sec. 1.1.1, were characterized in terms of response linearity together with the system noise equivalent power (NEP). For this purpose, both a CW laser source operating at $660 \mathrm{~nm}$ and the LEDs used for the in vivo measurements (Sec. 1.1.2) were employed. To study the detector sensitivity upper limit and linearity, the signal was collected by a Keythley 236 source-meter unit in the laser configuration acquisition, whereas, in order to study the detector and system minimum sensitivity, the same configuration employed for in-vivo measurement was utilized (Sec. 1.1.2). In the first configuration, the pump light intensity was monitored by a calibrated photodetector (Newport, 1936-R) and it was varied by using a concave lens and by changing the lens/detector distance. In the second configuration, the pump light impinging on the SiPMs was kept constant at Pow $=2 \mathrm{pW}\left(10 \mathrm{pW} / \mathrm{cm}^{2}\right)$ while dark count variability was assessed as a function of sampling frequency of the system.

The dark count variability was estimated as the standard deviation (SD) of the signal over an integration time that was dependent on the sampling frequency.

SNR was measured relying on signal and dark count variability and NEP was estimated as follows:

$\mathrm{NEP}=\frac{\mathrm{Pow}}{\mathrm{SNR}}$.

SiPMs temperature was fixed by using a thermostatic stage. SNR was finally estimated in vivo at the distances employed by the optical probe.

\subsection{Commercial System}

The SiPMs-LEDs system was compared with a commercial fNIRS oxymeter through an in vivo experiment. The commercial NIR spectrometer from ISS (Imagent ${ }^{\mathrm{TM}}$, Champaign, Illinois) was employed.

The apparatus is a FD system equipped with 32 laser diodes ( $\sim 1 \mathrm{~mW}$ power, 16 emitting light at $690 \mathrm{~nm}$ and 16 at $830 \mathrm{~nm}$ ) and 4 PMTs (bias range: -300 : $-1000 \mathrm{~V}$ ). Both the laser diodes and PMTs are modulated in the radiofrequency range at slightly different frequencies $(110 \mathrm{MHz}$ for photodiodes and 110.5 MHz for PMTs). The two different frequencies allow for heterodyne detection of an electrical signal at 5-kHz modulation. The signal was digitalized and a windowed fast Fourier

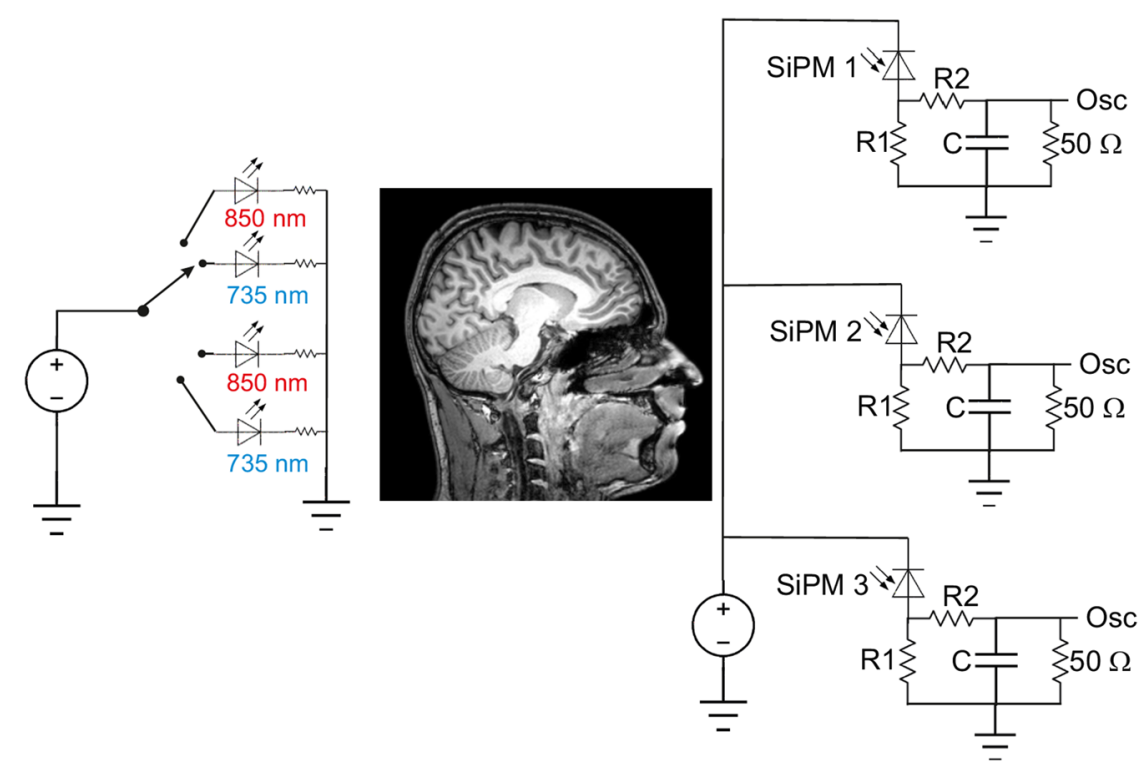

Fig. 3 Electrical scheme of the system. 
transform was applied over time. Only the DC component of the FFT was estimated in order to compare the results in a CW operation mode.

Four light sources (two injection point and two wavelengths) and three detectors were employed for the measurement. Timemultiplexing was employed for source coding with each source left on for $12.5 \mathrm{~ms}$, and a system sampling frequency of $20 \mathrm{~Hz}$. Light was sent to the scalp using multimodal optical fibers ( $0.4 \mathrm{~mm}$ core) and from the scalp back to the PMTs using fiber bundles ( $3 \mathrm{~mm}$ diameter). The fibers were held in place using soft, but rigid, custom-built optical patch with a same optical layout employed for the SiPMs-LEDs system that is depicted in Figs. 2 and 3.

\subsection{In-Vivo System Assessment}

\subsubsection{Measurement protocol}

After being informed about the methodologies and outcomes of the study, the subject provided informed consent to the measurements. The procedures used throughout the study were performed in agreement with the ethical standards of the Helsinki Declaration, 1964, and were approved by the local Human Board Review and Ethical Committee.

The recordings with the two systems were identical but asynchronous in time.

The subject (right-handed) C3 location (based on the 10 to 20 system) was identified and the optical patches are located, as reported in Fig. 6. The subject set comfortably on a chair and performed a right-hand finger tapping task locked to an auditory stimulation ("start," "stop" commands). The task was performed for four consecutive runs. Each run was constituted of $40 \mathrm{~s}$ of rest and alternating $20 \mathrm{~s}$ task and $20 \mathrm{~s}$ rest trials for four times. The procedure was repeated for the two different systems. The overall experiment involved 32 finger tapping tasks (16 per system).

\subsubsection{Data analysis}

The raw signal for each of the eight runs (four runs per apparatus) was extracted from the two fNIRS systems. While the commercial system already provided raw signals divided by source and detector, the in-house system required offline separation of each source contribution on each of the detector signals sampled at $10 \mathrm{KHz}$. Each of the four sources time course was extracted based on the time-multiplexing scheme by integrating each source signal during the "on" period. The final sample rate for the extracted signals was $20 \mathrm{~Hz}$, in accordance with the commercial system.

The following analysis was shared by the two systems and optical densities (ODs) were computed. OD is defined as follows:

$\mathrm{OD}=-\ln \left[\frac{\mathrm{I}(\mathrm{t})}{\mathrm{I}_{\mathrm{o}}}\right]$,

where $I(t)$ is the time dependence of the recorded signal intensity and $I_{\mathrm{o}}$ is its initial value.

A movement artifact correction procedure was further applied. ${ }^{48}$

In order to highlight brain hemodynamics frequencies, $0.2-\mathrm{Hz}$ low-pass and $0.01-\mathrm{Hz}$ high-pass, zero-lag, fifth order Butterworth digital filters were applied.
Variations in the concentration of oxyhemoglobin and deoxyhemoglobin were derived from each channel (two sources-one detector couple) based on the modified Lambert-Beer law: ${ }^{49}$

$$
\begin{aligned}
{\left[\begin{array}{c}
\mathrm{O}_{2} \mathrm{Hb} \\
\mathrm{HHb}
\end{array}\right]=} & \frac{1}{\rho}\left[\begin{array}{ll}
\varepsilon_{\mathrm{O}_{2} \mathrm{Hb}}\left(\lambda_{1}\right) \cdot \operatorname{DPF}\left(\lambda_{1}\right) & \varepsilon_{\mathrm{HHb}}\left(\lambda_{1}\right) \cdot \operatorname{DPF}\left(\lambda_{1}\right) \\
\varepsilon_{\mathrm{O}_{2} \mathrm{Hb}}\left(\lambda_{2}\right) \cdot \operatorname{DPF}\left(\lambda_{2}\right) & \varepsilon_{\mathrm{HHb}}\left(\lambda_{2}\right) \cdot \operatorname{DPF}\left(\lambda_{2}\right)
\end{array}\right]^{-1} \\
& \mathrm{X}\left[\begin{array}{l}
\mathrm{OD}\left(\lambda_{1}\right) \\
\mathrm{OD}\left(\lambda_{2}\right)
\end{array}\right]
\end{aligned}
$$

where $\mathrm{O}_{2} \mathrm{Hb}$ and $\mathrm{HHb}$ represent the changes in oxyhemoglobin and deoxyhemoglobin concentration, respectively, $\rho$ is the interoptode distance, $\varepsilon$ and DPF are, respectively, the extinction coefficients for the two chromophores and the differential pathlength factors at the wavelengths of interest $\left(\lambda_{1}\right.$ and $\left.\lambda_{2}\right)$. The extinction coefficients of the two forms of hemoglobin at the different wavelengths (four wavelengths, two per system) were extracted from Zijlstra et al. ${ }^{50}\left(\varepsilon_{\mathrm{O} 2 \mathrm{Hb}, 735 \mathrm{~nm}}=0.014 \mathrm{~mm}^{-1}\right.$, $\varepsilon_{\mathrm{O} 2 \mathrm{Hb}, 850 \mathrm{~nm}}=0.025 \mathrm{~mm}^{-1}, \quad \varepsilon_{\mathrm{HHb}, 735 \mathrm{~nm}}=0.039 \mathrm{~mm}^{-1}$, $\varepsilon_{\mathrm{HHb}, 850 \mathrm{~nm}}=0.019 \mathrm{~mm}^{-1}, \quad \varepsilon_{\mathrm{O} 2 \mathrm{Hb}, 690 \mathrm{~nm}}=0.0096 \mathrm{~mm}^{-1}$, $\varepsilon_{\mathrm{O} 2 \mathrm{Hb}, 830 \mathrm{~nm}}=0.021 \mathrm{~mm}^{-1}, \quad \varepsilon_{\mathrm{HHb}, 690 \mathrm{~nm}}=0.05 \mathrm{~mm}^{-1}$, and $\varepsilon_{\mathrm{HHb}, 830 \mathrm{~nm}}=0.017 \mathrm{~mm}^{-1}$ ). The DPFs were derived by Scholkmann \& Wolf $^{51}\left(\mathrm{DPF}_{735 \mathrm{~nm}}=6, \mathrm{DPF}_{850 \mathrm{~nm}}=5.5\right.$, $\mathrm{DPF}_{690 \mathrm{~nm}}=6.5$, and $\left.\mathrm{DPF}_{830 \mathrm{~nm}}=5.5\right)$.

For each finger tapping trial, $\mathrm{O}_{2} \mathrm{Hb}$ and $\mathrm{HHb}$ responses were selected from 10 seconds prior to the task up to $10 \mathrm{~s}$ after the task and baseline corrected based on the rest period before the task. 16 trials (4 per runs) were obtained for $\mathrm{O}_{2} \mathrm{Hb}$ and $\mathrm{HHb}$ in each channel for each system, and average responses and related variabilities were assessed. In order to compare the hemodynamic estimates between the two systems, the covariance metrics between average $\mathrm{O}_{2} \mathrm{Hb}$ and $\mathrm{HHb}$ with a standard hemodynamic response to the task were assessed ${ }^{48,52}$ [Fig. 7(a)]. The covariance metric allowed summarizing information of both shape and intensity of hemodynamic response for each channel, hemoglobin form and system. The comparison between the two systems was performed via a correlation analysis and a BlandAltman plot of the covariance metrics considering channel and hemoglobin forms as samples. ${ }^{53,54}$

\section{Results}

\subsection{System Characterization}

The first configuration allowed us to study SiPMs linearity and to obtain an upper limit of the measurable optical power. Figure 1(b) reports the average SiPMs photocurrent as a function of optical intensity measured at $25^{\circ} \mathrm{C}$ at 28,29 , and $30 \mathrm{~V}$, with an avalanche break down occurring at $27.7 \mathrm{~V}$. The apparent loss of linearity in the logarithmic plot for small optical intensity clearly depended on the offset introduced by the dark count rate (black circles). In fact, by subtracting the dark count rate [Fig. 1(c)], the linearity range increased. The upper limit was caused by the SiPMs pixel dead time related to the bias recharge after avalanche, which is limited by the RC constant of the quenching resistor of about $100 \mathrm{~ns}^{36}$

The lower limit of the SiPMs and system sensitivity (NEP) was measured with the recording apparatus and configuration employed in-vivo (second configuration).

Figure 4(a) reports the average SNR obtained with an incident light on the detectors of $2 \mathrm{pW}\left(10 \mathrm{pW} / \mathrm{cm}^{2}\right)$, whereas Fig. 4(b) reports the estimated NEP, both as a function of the measurement frequency. Data show an NEP between 0.8 and 

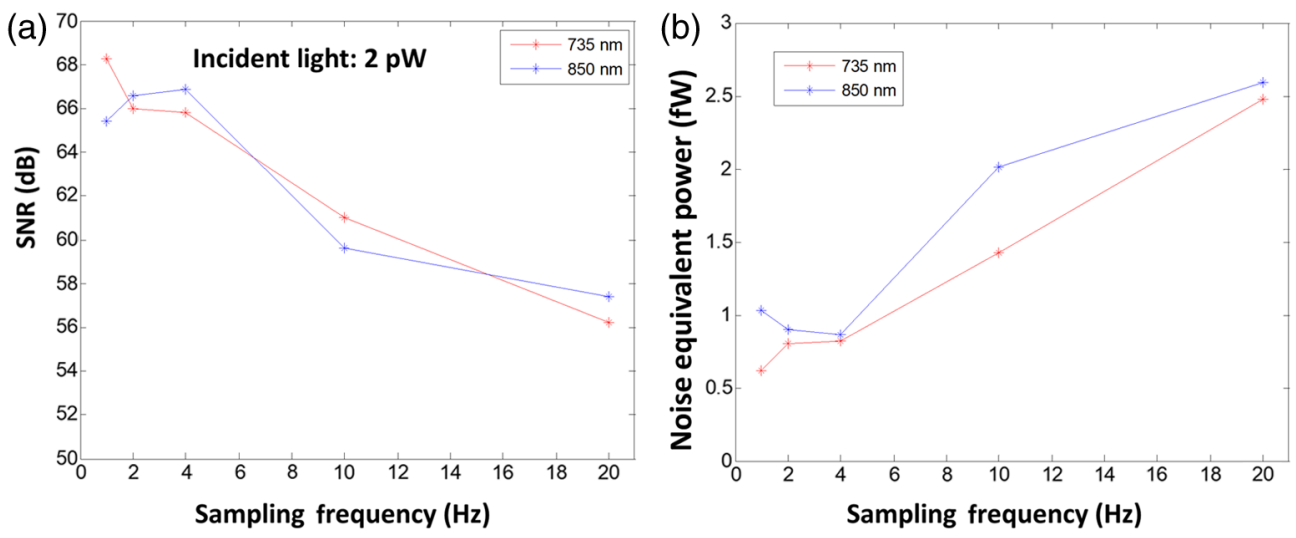

Fig. 4 (a) Average SNR of the system at a fixed incident light power of $2 \mathrm{pW}$ as a function of the sampling frequency of the system. (b) Estimated system's NEP as a function of the sampling frequency. Data were collected in a range (1 to $20 \mathrm{~Hz}$ ) compatible with actual fNIRS measurements.

$-2.5 \mathrm{fW}\left(5 \times 10^{-15} \mathrm{~W} / \mathrm{cm}^{2}\right.$ to $1.6 \times 10^{-14} \mathrm{~W} / \mathrm{cm}^{2}$ when considering SiPMs surface area) for both wavelengths $(10-\mathrm{kHz}$ recording bandwidth). Although slightly depending on wavelength and sampling frequency employed, the estimated system NEP was below $3 \mathrm{fW}$.

Figure 5 reports an example of signals from $1 \mathrm{LED}$ at $850 \mathrm{~nm}$ recorded by the three SIPMs. Data were taken in vivo at rest, and they were estimated with an integration time of $10 \mathrm{~ms}$. The average current was subtracted to expose physiological signals, such as heart rate and Mayer waves. ${ }^{23}$ These signals cause similar optical modulations when compared to the BOLD effect. The high SNR is evident. SNR was also estimated directly in vivo with LEDs multiplexing at $20 \mathrm{~Hz}$ and interoptode distances described above. Although fNIRS SNR may vary substantially depending on the regional tissue transparency, the interoptode distance, the presence of hairs, etc., at $10 \mathrm{kHz}$ sampling frequency (which is the frequency used during the task reported in this work), the average SNR of the SiPMs resulted to be $\sim 65 \mathrm{~dB}$.

\subsection{In-Vivo System Assessment}

Average $\mathrm{O}_{2} \mathrm{Hb}$ and $\mathrm{HHb}$ responses to the motor task and related standard errors for the six channels employed and the two

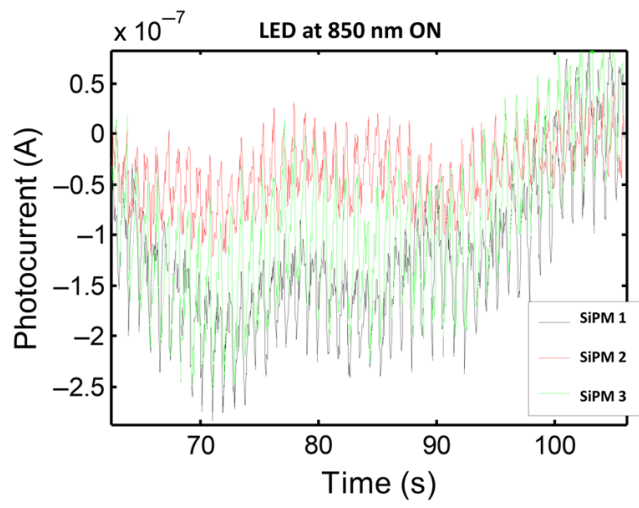

Fig. 5 Example of signals from $1 \mathrm{LED}$ at $850 \mathrm{~nm}$ recorded by the three SIPMs of the optical probe employed. The data were taken in vivo and at rest, and they were estimated with an integration time of $10 \mathrm{~ms}$. The average current was subtracted to expose physiological signals, such as heart rate and Mayer waves. systems are reported in Fig. 6. The task period is identified in time by the two vertical black lines. Clear similarities are visible between the estimated hemoglobin responses of the two systems. Notably, the short interoptode distance channel (Ch. 4), the one theoretically minorly sensitive to brain hemodynamic response, was the one with the smallest hemoglobin variations when compared to baseline for both systems.

However, because of the asynchronous measurements and the intrinsic variability of the physiological signal recorded, the hemoglobin responses between the two systems were not identical. In order to investigate similarities/dissimilarities between the two systems estimated BOLD responses, the metric of covariance between a standard hemodynamic response and the measured hemoglobin responses to the task was employed [Fig. 7(a)]. The absolute covariances obtained for each system (6 from $\mathrm{O}_{2} \mathrm{Hb}$ and $6 \mathrm{HHb}, 12$ total) were compared through a correlation analysis and a Bland-Altman plot. Figure 7(b) reports the scatterplot of covariances for the six channels and the two forms of hemoglobin (12 points) considering the two systems. A high correlation was found $\left(r^{2}=0.84, p<0.01\right)$.

Figure 7(c) reports the Bland-Altman plot of covariances for the two systems. The differences in the covariance metric are reported as a function of the average of the covariance metric. Importantly, no significance distance from 0 was found for the average difference $(p=0.66)$. Moreover, no points above and below 1.96 SD were found (1.96 SD considers a confidence intervals of $95 \%$ ), indicating no significant effect of the average response on the difference in responses between systems.

\section{Discussion}

fNIRS is increasing its popularity as a neuroimaging procedure. ${ }^{1}$ fNIRS historically relies on optical fibers to bring and collect light to and from the scalp. This characteristic provides the advantage of keeping the optical instrumentation away from the measurement, electrically isolating the subject. Moreover, it allows employing highly sensitive light detectors, such as PMTs (which are bulky and work at high voltages).

Because light sensitivity decays exponentially (within few centimeters) from the source-detector couple, fNIRS has the advantage to provide local information regarding brain activity, especially when compared to other scalp-based neuroimaging modalities, such as EEG or MEG. ${ }^{21}$ However, this aspect makes the technology dependent on multiple optodes (sources and detectors) at multiple interoptode distances when accurate 
Ch. 1
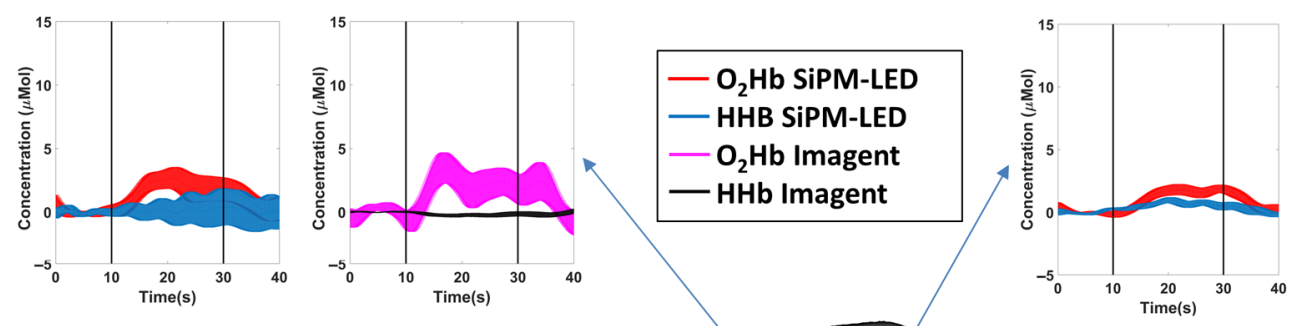

Ch. 4

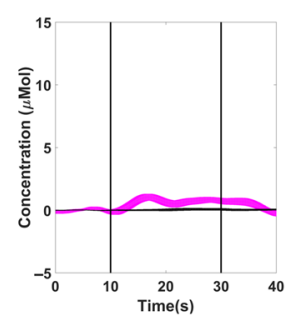

Ch. 5

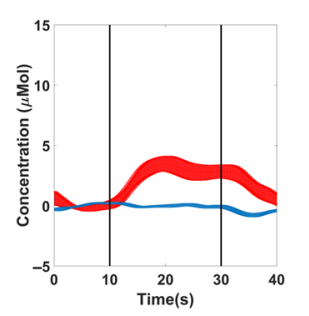

Ch. 2
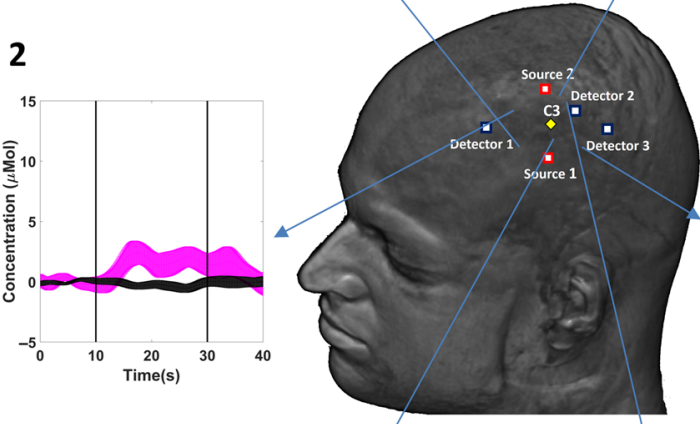

Ch. 3
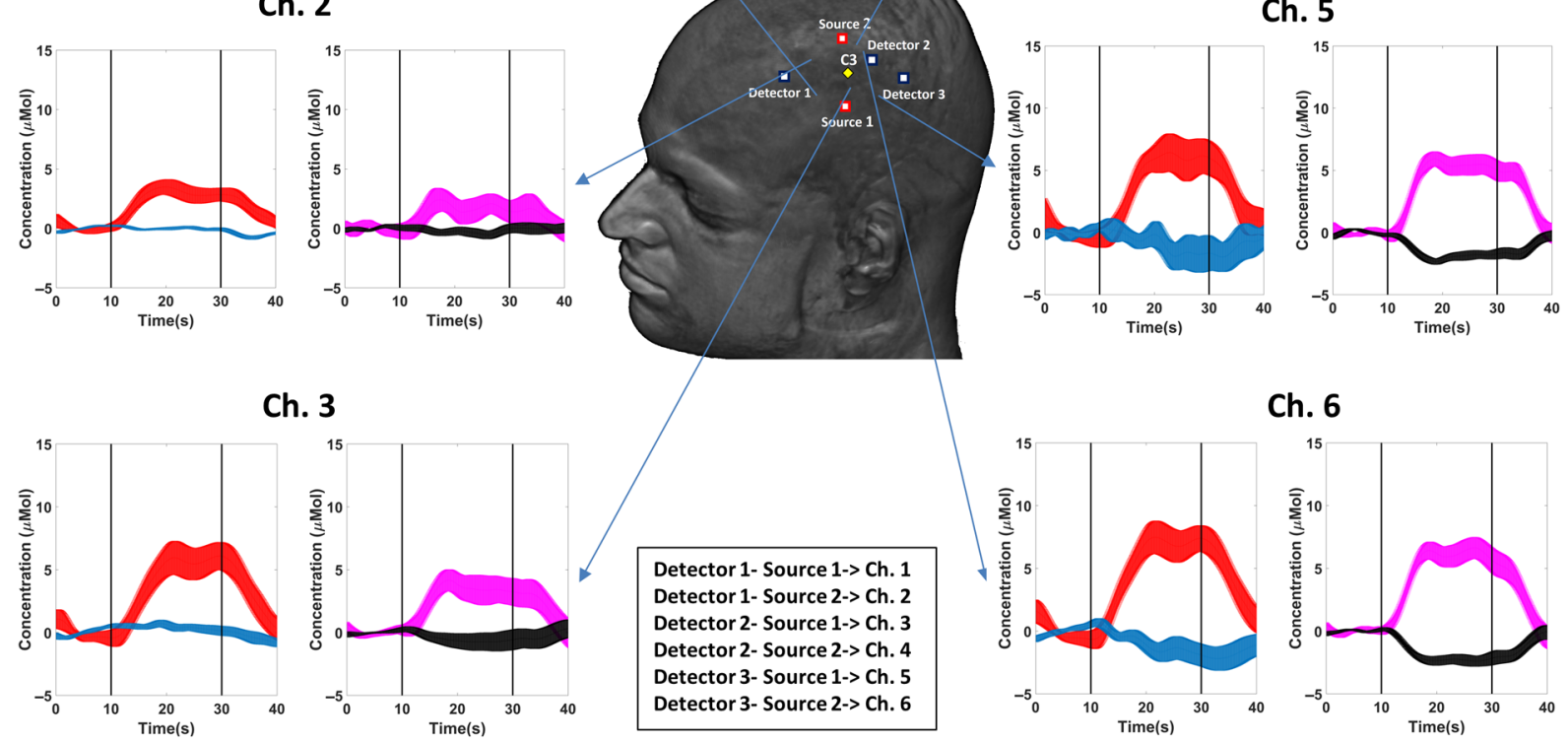

Ch. 6
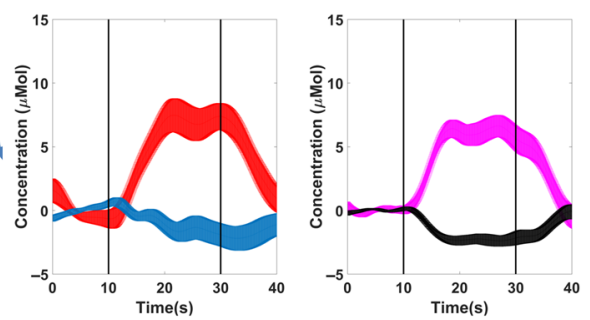

Fig. 6 Average $\mathrm{O}_{2} \mathrm{Hb}$ and $\mathrm{HHb}$ responses to the motor task and related standard errors for the six channels and the two systems employed. The task period is identified in time by the two vertical black lines. Optodes locations on the scalp together with subject's C3 location (10 to 20 system) are reported on a rendition of the subject's magnetic resonance image. The blue arrows connect the midpoint of the optical channels on the rendered magnetic resonance image to their estimate of $\mathrm{O}_{2} \mathrm{Hb}$ and $\mathrm{HHb}$ responses.

investigation of the region of interest is required. Multiple optodes at multiple (short and long, from $\sim 1.5 \mathrm{~cm}$ up to $\sim 6 \mathrm{~cm}$ in adults) distances are required for fNIRS in order to estimate effects of scalp related hemodynamic, to create a decent coverage of the brain cortex, and possibly to perform volumetric image reconstruction (DOT). In fact, fNIRS is definitely moving toward high-density optode layouts for neuroimaging investigation. ${ }^{24}$ However, a high number of fibers and fiber bundles increases the difficulties to provide a good optode to scalp coupling and it inevitably causes a loss in the lightweight properties of fNIRS, often restricting its usage to a laboratory environment.

A possible solution to these problems is to place sources and detectors directly on the scalp transferring the analog signal via thin cables to the acquisition system and avoiding optical fibers. Semiconductor light detectors are suitable for such a purpose. State-of-the-art wearable CW-fNIRS systems employ LEDs for light probing and photodiodes for light detection. However, photodiodes sensitivity and dynamic range are poor and, considering that light decays in tissue $\sim 1$ decade $/ \mathrm{cm}$, wearable systems are generally limited to a few sparse optodes at fixed source-detector distances. ${ }^{29-31}$

Highly sensitive semiconductor light detectors, such as SiPM, ${ }^{55}$ directly located on the scalp, could be suitable for future high-density, accurate and lightweight, fNIRS systems.
We presented and characterized a multichannel, multidistance, CW-fNIRS optical probe (Figs. 2 and 3) that relied on multiple SiPMs (three detectors, STMicroelectronics), and multiple LEDS (four sources, two injection points, two sources for each injection location at $735 \mathrm{~nm}, 850-\mathrm{nm}$ wavelengths) for a total of six fNIRS channels. The probe provided a limited number of optodes, but, since the optical patch was limited in space, it encompassed the required characteristics of a highdensity multidistance CW-fNIRS system and it can be easily expanded.

Notice that, although proof-of-concept of SiPM-based CWfNIRS systems was provided before, ${ }^{34,36,55}$ to the best of our knowledge, this is the first report of a multichannel, source time-multiplexed, SiPM-based CW-fNIRS system employed for an actual in vivo hemodynamic brain activity measurements.

The SiPMs were first characterized in linearity and dynamic range using two controlled experiment (Figs. 1 and 4). They showed an upper sensitivity limit of $10^{-6} \mathrm{~W} / \mathrm{cm}^{2}$ with a minimum sensitivity limit when coupled with the acquisition system of $10^{-14} \mathrm{~W} / \mathrm{cm}^{2}$ (NEP below $3 \mathrm{fW}$ considering the detector area) at a low operating voltage of $\sim 30 \mathrm{~V}$. This dynamic range would allow spanning interoptode distances of many centimeters.

NEP value measured in this work is much lower than other state-of-the-art wearable fNIRS systems ${ }^{56,57}$ (NEP of $\sim 400 \mathrm{fW}$ ). 

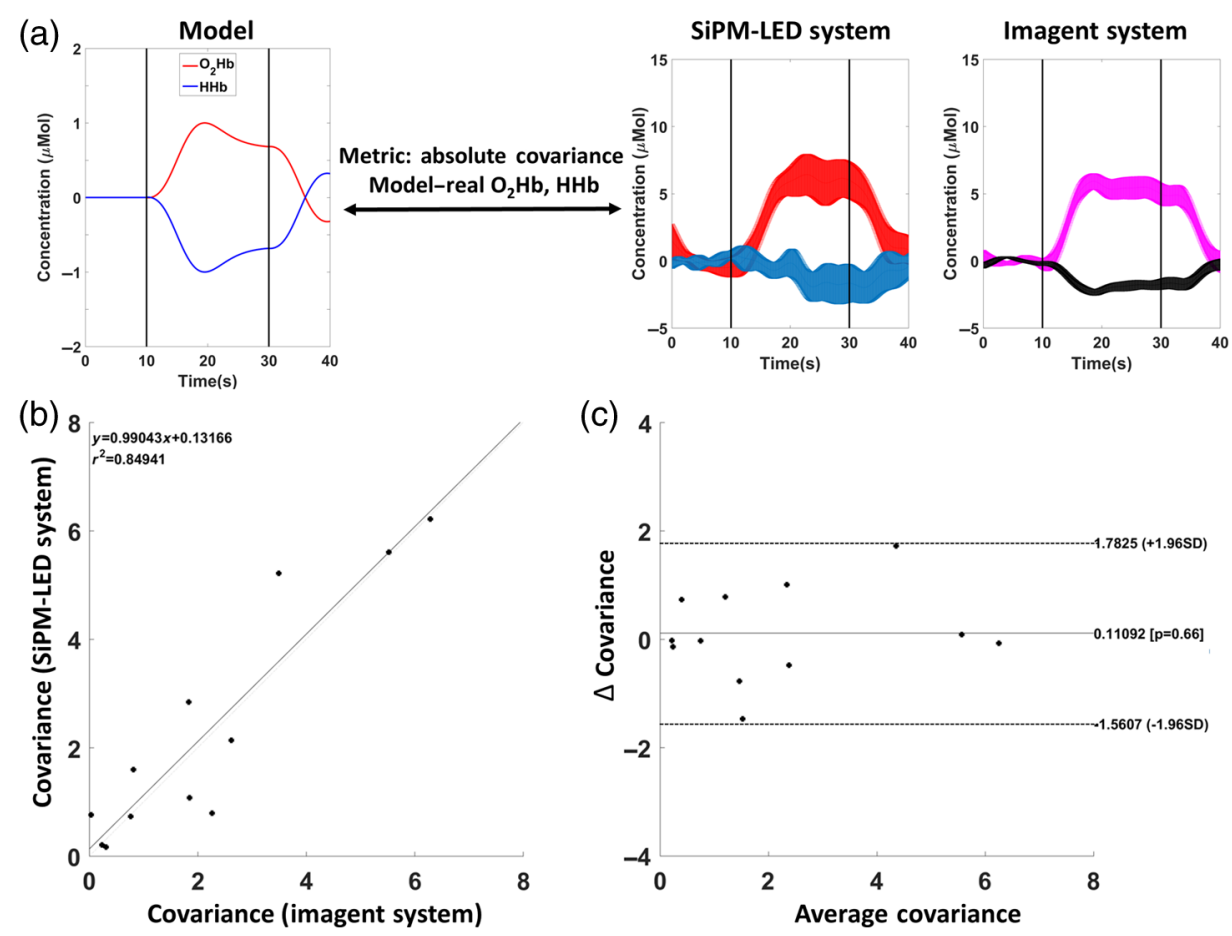

Fig. 7 (a) Schematization of the metric employed for system comparison. The metric of covariance between a standard hemodynamic response (left image) and the measured hemoglobin responses (right image) to the task was employed. (b) Scatterplot of covariances for the six channels and the two forms of hemoglobin (12 points) for one system (SiPM-LED) as a function of covariances of the commercial system (ISS Imagent ${ }^{\mathrm{TM}}$, Champaign, Illinois). (c) Bland and Altman plot of covariances of the two systems (SiPM-LED, ISS Imagent ${ }^{\mathrm{TM}}$ ). The differences in the covariance metric are reported as a function of the average of the covariance metric.

NEP improvement of two orders of magnitude is directly related to the higher SiPM sensitivity when compared to standard photodiodes (or APDs) in the wavelength range of interest (700 to $850 \mathrm{~nm}$ ).

These results were obtained with a highly flexible and lightweight probe due to the small $\left(\sim 1 \mathrm{~cm}^{2}\right)$ boards required for LEDs and SiPMs.

It should be highlighted that the operating voltage of the SiPM $(30 \mathrm{~V})$ may create some electrical safety issues, inducing a current of a few $\mathrm{mA}$ if put in contact with the skin. In order to avoid electrical contact with the skin, both the SiPMs and the LEDs boards were electrically isolated (refer to Sec. 1.1.1). Although conductive paths may arise in the insulator over time, the resistance of these paths rarely drops below the $\mathrm{k} \Omega$ ranges, still preventing significant current flowing in the skin. In theory, although not directly implemented in the system reported in this manuscript, a surge protector may also be employed.

The soft and wearable optical probe was further tested in vivo at rest, where the system showed recording capabilities of signals, such as pulse modulation and Mayer waves, which, based on the similar intensity between these signals and the fNIRS BOLD effect, proved the recording capabilities of the system (Fig. 5) with an estimated SNR of $\sim 65 \mathrm{~dB}$ at the interoptode distances employed.

Importantly, the system was tested and compared to a commercial fiber-based system (ISS Imagent ${ }^{\mathrm{TM}}$, Champaign, Illinois) in the retrieval of the hemodynamic oscillations in response to a motor task (Fig. 6). Using the metric of covariance between an ideal hemodynamic response to the task, which was extracted from the literature, ${ }^{52}$ and the hemodynamic responses extracted using the two different systems, a correlation analysis and a Bland-Altman plot showed clear similarities between the SiPM-LED and the Imagent system (Fig. 7), with no significant differences in the brain signals retrieved.

The results clearly showed the capabilities of SiPM sensors for fNIRS imaging and their possibility to represent future stateof-the-art detectors for flexible high-density optical neuroimaging. In fact, although the system reported here provided a limited number of optodes, it can be easily expanded relying on small LED and SiPM boards $\left(\sim 1 \mathrm{~cm}^{2}\right)$ that require only a cable connection to the acquisition system.

Scalp located light sources and highly sensitive semiconductor detectors may be of great help for expanding fNIRS as a neuroimaging tool and for integrating fNIRS with other scalp-based neuroimaging modalities, such as EEG, maintaining the characteristics of cheap, lightweight, and portable technology.

\section{Disclosures}

No conflicts of interest, financial or otherwise, are declared by the authors.

\section{Acknowledgments}

This study was partially funded by grant: 692470, H2020, ECSEL-04-2015-Smart Health, Advancing Smart Optical Imaging and Sensing for Health (ASTONISH). 


\section{References}

1. M. Ferrari and V. Quaresima, "A brief review on the history of human functional near-infrared spectroscopy (fNIRS) development and fields of application," Neuroimage 63(2), 921-935 (2012).

2. A. Villringer and B. Chance, "Non-invasive optical spectroscopy and imaging of human brain function," Trends Neurosci. 20(10), 435-442 (1997).

3. P. Giacometti and S. G. Diamond, "Correspondence of electroencephalography and near-infrared spectroscopy sensitivities to the cerebral cortex using a high-density layout," Neurophotonics 1(2), 025001 (2014).

4. M. E. Raichle, "Behind the scenes of functional brain imaging: a historical and physiological perspective," Proc. Natl. Acad. Sci. U. S. A. 95(3), 765-772 (1998).

5. G. Gratton, A. M. Chiarelli, and M. Fabiani, "From brain to blood vessels and back: a noninvasive optical imaging approach," Neurophotonics 4(3), 031208 (2017).

6. T. Farroni et al., "Infant cortex responds to other humans from shortly after birth," Sci. Rep. 3, 2851 (2013).

7. M. Costantini et al., "Studying social cognition using near-infrared spectroscopy: the case of social Simon effect," J. Biomed. Opt. 18(2), 025005 (2013).

8. M. Fabiani et al., "Neurovascular coupling in normal aging: a combined optical, ERP and fMRI study," Neuroimage 85(Pt 1), 592-607 (2014).

9. A. J. Fallgatter and W. K. Strik, "Right frontal activation during the continuous performance test assessed with near-infrared spectroscopy in healthy subjects," Neurosci. Lett. 223(2), 89-92 (1997).

10. A. Gallagher et al., "Near-infrared spectroscopy as an alternative to the Wada test for language mapping in children, adults and special populations," Epileptic Disord. 9(3), 241-255 (2007).

11. T. Grossmann et al., "Early cortical specialization for face-to-face communication in human infants," Proc. R. Soc. B 275(1653), 2803-2811 (2008).

12. S. Lloyd-Fox, A. Blasi, and C. E. Elwell, "Illuminating the developing brain: the past, present and future of functional near infrared spectroscopy," Neurosci. Biobehav. Rev. 34(3), 269-284 (2010).

13. M. Mahmoudzadeh et al., "Syllabic discrimination in premature human infants prior to complete formation of cortical layers," Proc. Natl. Acad. Sci. U. S. A. 110(12), 4846-4851 (2013).

14. N. Roche-Labarbe et al., "NIRS-measured oxy- and deoxyhemoglobin changes associated with EEG spike-and-wave discharges in children," Epilepsia 49(11), 1871-1880 (2008).

15. E. Watanabe et al., "Noninvasive cerebral blood volume measurement during seizures using multichannel near infrared spectroscopic topography," J. Biomed. Opt. 5(3), 287-290 (2000).

16. A. M. Chiarelli et al., "Fast optical signal in visual cortex: improving detection by general linear convolution model," Neuroimage 66, 194202 (2013).

17. A. M. Chiarelli, G. L. Romani, and A. Merla, "Fast optical signals in the sensorimotor cortex: general linear convolution model applied to multiple source-detector distance-based data," Neuroimage 85(Pt 1), 245-254 (2014)

18. A. D. Mora et al., "Towards next-generation time-domain diffuse optics for extreme depth penetration and sensitivity," Biomed. Opt. Express 6(5), 1749-1760 (2015)

19. B. Chance et al., "Phase modulation system for dual wavelength difference spectroscopy of hemoglobin deoxygenation in tissues," Proc. SPIE 1204, 481-491 (1990).

20. F. Scholkmann et al., "A review on continuous wave functional nearinfrared spectroscopy and imaging instrumentation and methodology," Neuroimage 85(Part 1), 6-27 (2014).

21. A. M. Chiarelli et al., "Combining energy and Laplacian regularization to accurately retrieve the depth of brain activity of diffuse optical tomographic data," J. Biomed. Opt. 21(3), 036008 (2016).

22. Y. Hoshi and M. Tamura, "Dynamic multichannel near-infrared optical imaging of human brain activity," J. Appl. Physiol. 75(4), 1842-1846 (1993).

23. I. Tachtsidis et al., "Investigation of cerebral haemodynamics by nearinfrared spectroscopy in young healthy volunteers reveals posturedependent spontaneous oscillations," Physiol. Meas. 25(2), 437-445 (2004).
24. A. T. Eggebrecht et al., "Mapping distributed brain function and networks with diffuse optical tomography," Nat. Photonics 8(6), 448-454 (2014).

25. A. P. Gibson, J. C. Hebden, and S. R. Arridge, "Recent advances in diffuse optical imaging," Phys. Med. Biol. 50(4), R1-43 (2005).

26. N. M. Gregg et al., "Brain specificity of diffuse optical imaging: improvements from superficial signal regression and tomography," Front. Neuroenerg. 2, 14 (2010).

27. A. M. Chiarelli et al., "Comparison of procedures for co-registering scalp-recording locations to anatomical magnetic resonance images," J. Biomed. Opt. 20(1), 016009 (2015).

28. A. von Luhmann et al., "M3BA: a mobile, modular, multimodal biosignal acquisition architecture for miniaturized EEG-NIRS based hybrid BCI and monitoring," IEEE Trans. Biomed. Eng. 64, 1199-1210 (2016).

29. T. Muehlemann, D. Haensse, and M. Wolf, "Wireless miniaturized invivo near infrared imaging," Opt. Express 16(14), 10323-10330 (2008).

30. P. Pinti et al., "Using fiberless, wearable fNIRS to monitor brain activity in real-world cognitive tasks," J. Visualized Exp. 106, e53336 (2015).

31. S. K. Piper et al., "A wearable multi-channel fNIRS system for brain imaging in freely moving subjects," Neuroimage 85(Pt 1), 64-71 (2014).

32. P. Buzhan et al., "Silicon photomultiplier and its possible applications," Nucl. Instrum. Methods Phys. Res. Sect. A 504(1-3), 48-52 (2003).

33. M. F. Santangelo et al., "Si photomultipliers for bio-sensing applications," IEEE J. Sel. Top. Quantum Electron. 22(3), 335-341 (2016).

34. R. Zimmermann et al., "Silicon photomultipliers for improved detection of low light levels in miniature near-infrared spectroscopy instruments," Biomed. Opt. Express 4(5), 659-666 (2013).

35. R. Re et al., "Probe-hosted silicon photomultipliers for time-domain functional near-infrared spectroscopy: phantom and in vivo tests," Neurophotonics 3(4), 045004 (2016).

36. R. Pagano et al., "Improvement of sensitivity in continuous wave near infra-red spectroscopy systems by using silicon photomultipliers," Biomed. Opt. Express 7(4), 1183-1192 (2016).

37. E. Sciacca et al., "Arrays of Geiger mode avalanche photodiodes," IEEE Photonics Technol. Lett. 18(15), 1633-1635 (2006).

38. P. Finocchiaro et al., "Characterization of a novel 100-channel silicon photomultiplier-part II: charge and time," IEEE Trans. Electron Devices 55, 2765-2773 (2008).

39. M. Mazzillo et al., "Timing performances of large area silicon photomultipliers fabricated at STMicroelectronics," IEEE Trans. Nucl. Sci. 57(4), 2273-2279 (2010).

40. M. Mazzillo et al., "Electro-optical performances of p-on-n and n-on-p silicon photomultipliers," IEEE Trans. Electron Devices 59(12), 34193425 (2012)

41. M. Mazzillo et al., "Silicon photomultiplier technology at STMicroelectronics," IEEE Trans. Nucl. Sci. 56(4), 2434-2442 (2009).

42. www.hamamatsu.com

43. www.sensl.com.

44. www.ketek.net.

45. G. Adamo et al., "Responsivity measurements of N-on-P and P-on-N silicon photomultipliers in the continuous wave regime," 8629, 86291A (2013).

46. R. Pagano et al., "Dark current in silicon photomultiplier pixels: data and model," IEEE Trans. Electron Devices 59(9), 2410-2416 (2012).

47. M. Mazzillo et al., "Noise reduction in silicon photomultipliers for use in functional near-infrared spectroscopy," IEEE Trans. Radiat. Plasma Med. Sci. 1(3), 212-220 (2017).

48. A. M. Chiarelli et al., "A kurtosis-based wavelet algorithm for motion artifact correction of fNIRS data," Neuroimage 112, 128-137 (2015).

49. D. T. Delpy et al., "Estimation of optical pathlength through tissue from direct time of flight measurement," Phys. Med. Biol. 33(12), 1433-1442 (1988).

50. W. G. Zijlstra, A. Buursma, and W. P. Meeuwsen-van der Roest, "Absorption spectra of human fetal and adult oxyhemoglobin, deoxyhemoglobin, carboxyhemoglobin, and methemoglobin," Clin. Chem. 37(9), 1633-1638 (1991).

51. F. Scholkmann and M. Wolf, "General equation for the differential pathlength factor of the frontal human head depending on wavelength and age," J. Biomed. Opt. 18(10), 105004 (2013).

52. W. D. Penny et al., Statistical Parametric Mapping: The Analysis of Functional Brain Images, Academic Press, London, United Kingdom (2011). 
53. J. M. Bland and D. G. Altman, "Measuring agreement in method comparison studies," Stat. Methods Med. Res. 8(2), 135-160 (1999).

54. D. Formenti et al., "Skin temperature evaluation by infrared thermography: comparison of two image analysis methods during the nonsteady state induced by physical exercise," Infrared Phys. Technol. 81, 32-40 (2017).

55. D. Sanfilippo et al., "Design and development of a fNIRS system prototype based on SiPM detectors," 8990, 899016 (2014).

56. J. K. Choi et al., "Time-divided spread-spectrum code-based $400 \mathrm{fW}-$ detectable multichannel fNIRS IC for portable functional brain imaging," IEEE J. Solid-State Circuits 51(2), 484-495 (2016).

57. D. Chitnis et al., "Functional imaging of the human brain using a modular, fibre-less, high-density diffuse optical tomography system," Biomed. Opt. Express 7(10), 4275-4288 (2016).

Antonio M. Chiarelli received his BS and MS degrees in physics engineering and optics and photonics from the Polytechnic University of Milan, Italy, in 2006 and 2009, respectively. In 2013, he received his $\mathrm{PhD}$ in neuroimaging technologies from the University of G. D'Annunzio in Chieti-Pescara, Italy. From 2013 to 2017, he was a postdoctoral researcher at Beckman Institute, University of Illinois at Urbana-Champaign. Since 2017, he is an assistant professor of physics applied to medicine and biology at Institute for Advanced Biomedical Technology (ITAB), at the University of Chieti-Pescara, Italy. His research interests focus on optical brain imaging methods, their integration with other neuroimaging technologies, model of physical systems and machine learning applied to the study of brain status and function.

Sebania Libertino joined the National Council of Research (CNR) in 1999. Since 2007, she is a senior researcher at the Instituto per la Microelettronica e Microsistemi (IMM). Her research interests include optical and electrical sensors for biomedical and environmental applications. She has coauthored more than 120 papers published on international journals and holds three patents all extended to USA.

Filippo Zappasodi is presently an associate professor of applied physics at the University of G. d'Annunzio, Chieti, Italy. Since 2001, he has participated in 13 national and international projects, which received grants from Government Agencies, European Union, and Private Foundations, on topics connected to neuroimaging, brain data analysis, and pathologies of the central nervous system. He is author of 80 scientific articles in international indexed journals, with about 2000 citations and an $\mathrm{H}$ index of 26.
Massimo Mazzillo received his MS degree in physics with honors from the University of Bari, Italy, in 2002. Since 2002, he has been with R\&D STMicroelectronics, Catania, where he currently works as senior staff technology development engineer at the design of silicon and semiconductor compound optical detectors. His research interests also include the development of environment, health, and biosensors. He filed 23 patents and authored about 100 papers published in peer-reviewed journals and conference proceedings.

Francesco Di Pompeo received his MS degree in physics from the University of L'Aquila, Italy, in 2005 and his PhD in physics in 2010 while working on the WArP experiment at the Gran Sasso National Laboratory, Italy. From 2010, he is a postdoctoral researcher at ITAB University of Chieti-Pescara, Italy. He was involved in the Human Connectome Project (2010-2016) for the development of analysis software of resting state data. He is now involved in the ASTONISH project for the development of smart optical sensing. He authored more than 15 papers published in peer-reviewed journals and conference proceedings.

Arcangelo Merla received his MS degree in physics from the University of Bologna, Italy, in 1994 and his $\mathrm{PhD}$ in biomedical technology from the University of Chieti-Pescara, Italy, in 2001. Since 2002, he is director of the Infrared Imaging Lab at ITAB at the University of Chieti-Pescara, where he is also an associate professor of physics applied to medicine and biology. He currently works at the development of applications of thermal IR imaging and fNIRS in neurosciences and human-machine interaction. He authored more than 130 papers published in peer-reviewed journals and conference proceedings.

Salvatore Lombardo joined the Italian National Research Council (CNR) in 1994. Since 2007, he is a research director of CNR at IMM. In his research, in the field of semiconductor devices, he has coordinated several national and European projects, and collaborations in USA, Singapore, and Israel. He is author of more than 250 scientific articles, with more than 4500 citations and $\mathrm{H}$ index of 31 .

Giorgio Fallica graduated in physics at the University of Catania in 1978. Since 1980, he joined STMicroelectronics, where he currently works in $R \& D$ department. During his experience in STMicroelectronics, he gained multidisciplinary competencies in the development of integrated devices for power electronics, DNA and protein sensors, single-photon detectors, nuclear particle detectors, and silicon light-emitting devices. He issued 13 international patents and more than 100 scientific papers and contributions to conferences. 